\title{
Amino Acid Precursors from Carbon Monoxide in Simulated Interstellar Dust Ice Mantle by UV Irradiation at $10 \mathrm{~K}$
}

\author{
Yoshinori Takano, Kentaro Ushio, Takeo Kaneko, Kensei Kobayashi, ${ }^{* \dagger}$ and Hirofumi Hashimoto ${ }^{\dagger \dagger}$ \\ Department of Chemistry and Biotechnology, Yokohama National University, 79-5 Tokiwadai, Hodogaya-ku, Yokohama $240-8501$ \\ ${ }^{\dagger}$ Institute of Space and Astronautical Science, 3-1-1 Yoshinodai, Sagamihara 229-8510 \\ ${ }^{\dagger}$ Institute of Engineering Mechanics, University of Tsukuba, Tennodai, Tsukuba 305-8573
}

(Received March 26, 2003; CL-030262)

An ice mantle mixture of carbon monoxide, ammonia (or dinitrogen), and water was irradiated in a highly vacuumed cryostat with ultraviolet light at $10 \mathrm{~K}$. The primary products were identified as amino acid precursors when ammonia was used as a nitrogen source, which strongly suggested abiotic formation of bioorganic compounds in ice mantle of interstellar dusts in molecular clouds. The present results have significant implication for organic formation in interstellar dust ice mantles and the energetics of nitrogen fixation.

Fundamental building blocks associated with life like amino acids should have been formed in extraterrestrial environments ${ }^{1}$ and delivered by comets and meteorites. ${ }^{2}$ Greenberg et al. ${ }^{3}$ proposed a cyclic evolutional model of interstellar dusts: Organic compounds were formed and transformed in interstellar dusts (ISDs) when they travel in molecular clouds and diffuse clouds, then they were preserved in comets when ISDs grew into comets in a protosolar system. Representative carbon sources are carbon monoxide, formaldehyde and methanol; the predominant nitrogen source is ammonia. ${ }^{3,4}$ Dinitrogen $\left(\mathrm{N}_{2}\right)$ may exist in a molecular cloud environment, but it cannot be detected spectrometrically. ${ }^{5}$ Those ices are irradiated with galactic cosmic rays and secondary photons of ultraviolet. As carbon sources, methanol and cyanide would plausibly be present in ISDs in dense clouds, but they have not been detected in ISDs in diffuse clouds. Briggs et al. ${ }^{6}$ suggested that glycine was formed at $12 \mathrm{~K}$ after $\mathrm{UV}$ irradiation of an ice mixture of carbon monoxide, methane, ammonia and water, but the experiments were not conducted quantitatively and no other amino acids except glycine were detected. Recent experimental approaches from mixtures containing methanol by UV irradiation ${ }^{7,8}$ and quantitative discussion ${ }^{9}$ were significant for molecular cloud organics. However, the reactional trigger in study of molecular cloud environments to form amino acid precursors has to be clarified for nitrogen sources as either ammonia or dinitrogen. Here we report the formation of amino acid precursors related with energetics of nitrogen fixation in a simulated ice mantle of ISDs in molecular clouds ( $10 \mathrm{~K})$ under highly vacuumed condition with ultraviolet photons.

A schematic view of the apparatus of the molecular cloud simulation experiment is shown in Figure 1. In order to simulate astrophysical conditions, a cryogenic experimental system ${ }^{10}$ was constructed to make an ice mantle of ISDs in molecular clouds and to irradiate them with UV. The UV photon source $\left(9.2 \times 10^{14} \mathrm{eV} / \mathrm{s}\right)$ used was $150-\mathrm{W}$ deuterium lamp equipped with an $\mathrm{MgF}_{2}$ window (Hamamatsu Photonics L 1835). A copper substrate located in the cryostat was cooled down to ca.

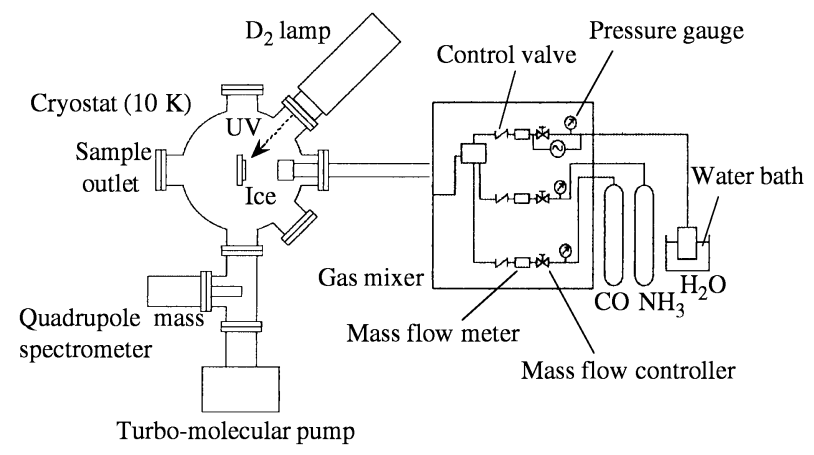

Figure 1. Schematic view of highly vacuumed and cryogenic system for UV irradiation experiment. $\mathrm{D}_{2}$ lamp used was newly purchased from Hamamatsu Photonics Co.

$10 \mathrm{~K}$ with a cold head (Iwatani Type D 105) and a helium compressor (Iwatani Type CW-301). The estimated thickness of the ice was $50 \mu \mathrm{m} .{ }^{1} \mathrm{~A}$ turbo molecular pump was used to evacuate the inside of the cryostat below $10^{-7}$ Torr. A gas mixer was composed of three mass flow controllers and high vacuum valves (KOFLOC, Japan) to make a gas mixture to be the desired mixing ratio. A quadrupole mass spectrometer (Balzers Model QMG-064) was used for analysis of volatile compounds. Carbon monoxide, ammonia and nitrogen of ultra-pure grade used as starting materials for irradiation experiments were purchased from Nihon Sanso Co. A gas mixture of carbon monoxide $\left(0.1 \mathrm{~cm}^{3} \cdot \mathrm{min}^{-1}\right)$, ammonia $\left(0.02 \mathrm{~cm}^{3} \cdot \mathrm{min}^{-1}\right)$ or nitrogen $\left(0.02 \mathrm{~cm}^{3} \cdot \mathrm{min}^{-1}\right)$ and water $\left(0.65 \mathrm{~cm}^{3} \cdot \mathrm{min}^{-1}\right)$ was constantly introduced in order to prepare molecular cloud ice mantles. Then, the ice mixtures were irradiated with UV photons $(\lambda>120 \mathrm{~nm})$ from the deuterium lamp at a rate of $9.2 \times 10^{14} \mathrm{eV} / \mathrm{s}(=0.53 \mathrm{~J} / \mathrm{h})$ for $108 \mathrm{~h}$. Blank runs were also performed in the same manner.

After irradiation, the temperature of the metal disk was naturally raised to room temperature at the rate of ca. $60 \mathrm{~K} \cdot \mathrm{h}^{-1}$, while generated gases were analyzed with a quadrupole mass spectrometer (ionization voltage: $100 \mathrm{eV} ; \mathrm{m} / z$ 1-64 were scanned for $128 \mathrm{~s}$ ) located between the cryostat and the turbo molecular pump. After analysis of volatile products, residual yellow-brown matter on the metal disk was recovered with water, which was subjected to amino acid analysis. Irradiation products in aqueous solution were acid-hydrolyzed with $6 \mathrm{~mol} \cdot \mathrm{dm}^{-3} \mathrm{HCl}$ at $383 \mathrm{~K}$ for $24 \mathrm{~h}$. After suitable procedures, determination of amino acids was performed. ${ }^{9}$

Corresponding mass peaks of hydrogen cyanide $(\mathrm{m} / \mathrm{z} 27)$, methanol $(m / z, 32)$ and ethanol $(m / z 46)$ were not observed while they were observed in the mass spectrum obtained in 


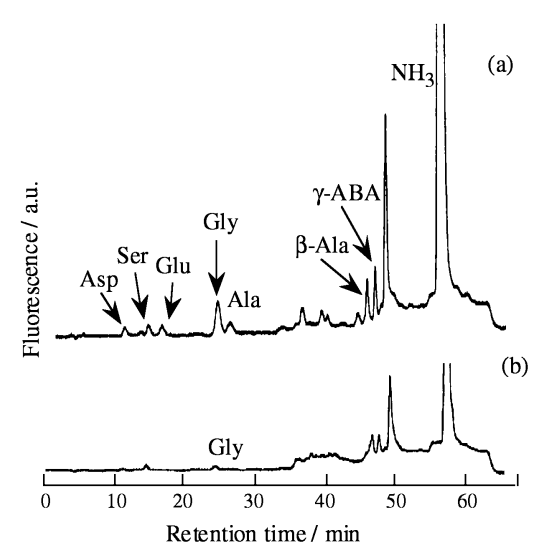

Figure 2. Ion-exchanged chromatogram of UV irradiated sample from the mixtures of (a) carbon monoxide-ammonia-water and, (b) carbon monoxide-ammonia, at $10 \mathrm{~K}$. The fraction was after acid hydrolysis. Abbreviations.Asp: aspartic acid, Ser: serine, Glu: glutamic acid, Gly: glycine, Ala: alanine, $\beta$-Ala: $\beta$-alanine, $\gamma$-ABA: $\gamma$-aminobutyric acid.

$3 \mathrm{MeV}$ proton irradiation experiments. ${ }^{1}$ The relative intensity of carbon monoxide was smaller than that of ammonia. Thus, carbon monoxide in the ice mantle was vaporized during irradiation.

As shown in Figure 2a, some proteinous amino acids such as glycine, alanine, aspartic acid, glutamic acid and non-proteinous amino acids such as $\beta$-alanine, $\gamma$-aminobutyric acid were determined in hydrolysates of the product. $\alpha$-Aminobutyric acid was trace amount in this experiment. The presence of non-proteinous amino acids and the procedural blank indicate that those products are not contaminated but were abiotically synthesized. Quantitative result of amino acid analysis was obtained as shown in Table 1 . The yield of glycine (161 pmol), the simplest amino acid, was predominant among the detected amino acids. ${ }^{10}$ An ice mixture of carbon monoxide and ammonia without water was also irradiated; however, only trace amount of amino acids could be detected. Hence it is shown that amino acid precursors could be formed at $10 \mathrm{~K}$ from such simple gas mixtures without methanol ${ }^{7,8}$ and hydrogen cyanide. ${ }^{7}$

When dinitrogen $\left(\mathrm{N}_{2}\right)$ was used as a nitrogen source, no amino acid was identified even after hydrolysis. Since dissociation of the nitrogen-nitrogen triple bond requires UV of much shorter wavelength than $127 \mathrm{~nm},{ }^{11}$ it could not be photo-dissociated with UV in this experiment. Carbon monoxide bond can be photo-dissociated only with UV of $111 \mathrm{~nm} .{ }^{11}$ If ammonia coexists, triggers derived from ammonia could contribute to the reaction, likewise photo-destruction of water molecules. ${ }^{12}$ The

Table 1. The amount of determined amino acids obtained from the gas mixtures of carbon monoxide, ammonia, and water at $10 \mathrm{~K}$. Total energy deposit was $3.5 \times 10^{20} \mathrm{eV}$.

\begin{tabular}{lc}
\hline Amino acids & Amount/pmol \\
\hline Glycine & 161 \\
Alanine & 81 \\
Serine & 43 \\
$\beta$-Alanine & 106 \\
$\gamma$-Aminobutylic acid & 116 \\
Aspartic acid & 41 \\
Glutamic acid & 54 \\
\hline
\end{tabular}

$\mathrm{C}-\mathrm{H}$ bond of methane $\left(\mathrm{CH}_{4}\right)$ would be photo-dissociated below $276 \mathrm{~nm},{ }^{11}$ and it also contribute to form organics in an ice mantle of ISDs in molecular clouds. ${ }^{6}$

It has been proved that primary irradiated products by particle energy sources (i.e. helium ion, ${ }^{13}$ proton, ${ }^{1,13}$ and electro$\mathrm{n}^{1,13}$ ) and photon sources (i.e. $\gamma$-rays, ${ }^{1,9}$ soft $\mathrm{X}$-rays, ${ }^{14}$ and $\mathrm{UV}^{9,15}$ ) were not free amino acids (monomer constituents), but amino acid precursors. ${ }^{16}$ In order to evaluate the yields of amino acids quantitatively, $G$-value (number of formed molecules per $100 \mathrm{eV}$ ) of glycine after acid-hydrolysis were discussed. ${ }^{9}$ The $G$-value of glycine formation under cryogenic highly vacuumed conditions in the present experiment was $2.7 \times 10^{-5}$. The gas mixture of the same composition gave the $G$-value of $2.2 \times 10^{-2}$ at ambient temperature and pressure. ${ }^{15}$ These results show a difference of three orders of magnitude. The reason seems to be that part of $\mathrm{CO}$ condensation might be not completed on the ice mixture. The $G$-value of the gas mixture of carbon monoxide, dinitrogen, and water was at least four orders of magnitude smaller than that when ammonia was used as a nitrogen source. ${ }^{15}$ Ammonia and coexisted water were easily photo-dissociated by UV photons and the resulting activated species can work as reaction triggers: Amino acid precursors could be, therefore, synthesized.

The authors would like to thank Dr. A. Kouchi, Hokkaido University and Dr. J. Koike, Tokyo Institute of Technology, for their experimental help. This research was supported in part by a Grant-In-Aid (No. 14340170) from MEXT (Ministry of Education, Culture, Sports, Science and Technology, Japan).

\section{References}

1 T. Kasamatsu, T. Kaneko, T. Saito, and K. Kobayashi, Bull. Chem. Soc. Jpn., 70, 1021 (1997).

2 C. F. Chyba and C. Sagan, Nature, 355, 125 (1992).

3 J. M. Greenberg and A. Li, Biol. Sci. Space, 12, 96 (1998).

4 H. Cottin, M. C. Gazeau, and F. Raulin, Planet. Space Sci., 47, 1141 (1999).

5 “Chronological Scientific Tables, 2001," ed. by National Astronomical Observatory of Japan, Tokyo (2001).

6 R. Briggs, G. Ertem, J. P. Ferris, J. M. Greenberg, P. J. Mcain, C. X. Mendoza-Gomes, and W. Shutte, Origins Life Evol. Biosphere, 22, 287 (1992).

7 M. P. Bernstein, J. P. Dworkin, S. A. Sandford, G. W. Cooper, and L. J. Allamandora, Nature, 416, 401 (2002).

8 G. M. Munoz Caro, U. J. Meierhenrich, W. A. Schutte, B. Barbier, A. A. Segavia, H. Rosenbauer, W. H. P. Thiemann, A. Brack, and J. M. Greenberg, Nature, 416, 403 (2002).

9 Y. Takano, H. Masuda, T. Kaneko, and K. Kobayashi, Chem. Lett., 2002, 986.

10 K. Kobayashi, T. Kaneko, A. Kouchi, H. Hashimoto, T. Saito, and M. Yamashita, Adv. Space Res., 23, 401 (1999).

11 H. Okabe, "Photochemistry of Small Molecules," Wiley-Interscience, New York (1978).

12 N. Watanabe, T. Horii, and A. Kouchi, Astrophys. J., 541, 772 (2000).

13 K. Kobayashi, T. Kaneko, T. Saito, and T. Ohima, Origins Life Evol. Biosphere, 28, 155 (1998).

14 J. Takahashi, T. Hosokawa, H. Masuda, T. Kaneko, K. Kobayashi, T. Saito, and Y. Utsumi, Appl. Phys. Lett., 74, 877 (1999).

15 Y. Takano, K. Ushio, H. Masuda, T. Kaneko, K. Kobayashi, J. Takahashi, and T. Saito, Anal. Sci., 17, 1635 (2001).

16 K. Kobayashi, H. Masuda, K. Ushio, A. Ohashi, H. Yamanashi, T. Kaneko, J. Takahashi, T. Hosokawa, H. Hashimoto, and T. Saito, Adv. Space Res., 27, 207 (2001). 